\title{
Experimental study of particle shape dependence of avalanches inside a ro- tating drum
}

\author{
Jian Chen ${ }^{1, *}$, Dominik Krengel ${ }^{2, * *}$, and Hans-Georg Matuttis ${ }^{3, * * *}$ \\ ${ }^{1}$ Japan Agency for Marine-Earth Science and Technology, 3173-25, Showa-machi, Kanazawa, Yokohama, Kanagawa 236-0001, Japan \\ ${ }^{2}$ Yokohama National University, Tokiwadai 79-5, Hodogaya, Yokohama, Kanagawa 240-8501, Japan \\ ${ }^{3}$ The University of Electro-Communications, 1 Chome-5-1, Chofugaoka, Chofu, Tokyo 182-8585, Japan
}

\begin{abstract}
We investigate the avalanches of spherical and non-spherical granular particles inside half-filled rotating drums. The time series of the center of gravity of the particle assemblies are obtained via image analysis and their single-sided amplitude (SSA) spectra are analyzed. The spectra features of this new indicator turn out to be characteristic for the avalanches, in terms of the existence of peaks in the low-frequency range and the decay rate of high frequency components. The SSA spectrum has a peak for the packings of non-spherical particles but not for the spherical particles. The high frequency part is characterized by a power law decay $1 / f^{a}(a>0)$. A $1 / f$-decay is found only for the spherical particles. For the packings of cornered particles, the exponents significantly deviate from $a=1$. As $1 / f$ spectra are often associated with self-organized criticality and therefore a scale invariance of the dynamics, we may conclude that there is no scale-invariant structure for granular avalanches. Considering the small number of particles and the regularity of convex particle shapes being used, the spectral features revealed in this study could be utilized for validating particle simulations.
\end{abstract}

\section{Introduction}

There exists a vast literature on granular avalanches due to the lasting interest on this "old" topic probably initiated by P. Bak, C. Tang and K. Wiesenfeld (BTW) [1] who argued that Self Organized Criticality (SOC) is the mechanism to explain the $1 / f$ noise (also referred as "flicker noise") abundant in nature, see e.g., the reviews [2, 3]. BTW used only a theoretical concept of sand piles and avalanches, to explain the basic idea of SOC, without considering any "real" granular avalanches.

The experiment on sand piles of spherical glass beads by Jaeger et al. [4] contradicted the SOC conjecture that the size distributions of avalanches of sand pile should follow a power law. For varieties of rice of different aspect ratio, Frette et al. [5] reported results which, depending on the kind (shape) of the rice grains, could be consistent with SOC or the opposite. Besides the (controversial) size distribution, other interesting aspects of granular avalanches have also attracted great attention, e.g., the transition from discrete to continuous avalanches [6,7], the formation and growth of avalanches [8,9], the relaxation dynamics [10], and various dynamic properties of avalanches [11-14].

In this paper, we study the avalanches of spherical and non-spherical particles inside a rotating drum to reveal the influence of particle shape on avalanche dynamics. Instead of conventional slope angles, we focus on a novel indicator, the variation in the center of gravity, for avalanches.

\footnotetext{
*e-mail: jchen@jamstec.go.jp

**e-mail: krengel-dominik-ds@ynu.ac.jp

***e-mail: hg@mce.uec.ac.jp
}

A video is available at https://doi.org/10.48448/c8wh-vw85

(C) The Authors, published by EDP Sciences. This is an open access article distributed under the terms of the Creative Commons Attribution License 4.0

(http://creativecommons.org/licenses/by/4.0/)
We show that the spectral features of this indicator can capture the characteristics of avalanches with different particle shapes. Our results can serve as reference data for validating particle simulations, for the regularity of particle shapes and the small particle numbers being used.

\section{Methodology}

\subsection{Experimental setup}

The experimental setup is shown in Fig. 1 (a): An acrylic cylindrical drum was driven by a motor (4IK25GN-SW2 with controller FE100A from ORIENTAL MOTOR corporation) while the depth $d$ of the drum along the axial direction was fixed. The videos were taken in axial direction by a CASIO EX-F1 camera.

Two drums were used to ensure the number of particles are large enough for statistical measurement: a large drum with inner radius of $r=96[\mathrm{~mm}]$ and a small one with $r=71.5$ [mm]. The depth of both drums was fixed to 65 [mm], about 4 polyhedral particles. The drum was half-filled with spherical particles or with acrylic polyhedra. One type of polyhedral particles has 14 corners and 24 faces and the other is cubic, as shown in Fig. 1 (b). Those polyhedral particles are of density $1200\left[\mathrm{~kg} / \mathrm{m}^{3}\right]$, Young's modulus $3.2[\mathrm{GPa}$ ] and friction coefficient about 0.4 to 0.5 (in-house measurement on inclined slopes). Spherical particles (beads for airsoft guns) of diameter $8[\mathrm{~mm}]$ were used. The spherical particles are made from ABS with surface coating, with a density of $1230\left[\mathrm{~kg} / \mathrm{m}^{3}\right]$, Young's modulus 6 [GPa]. About 2000 spherical particles and a 


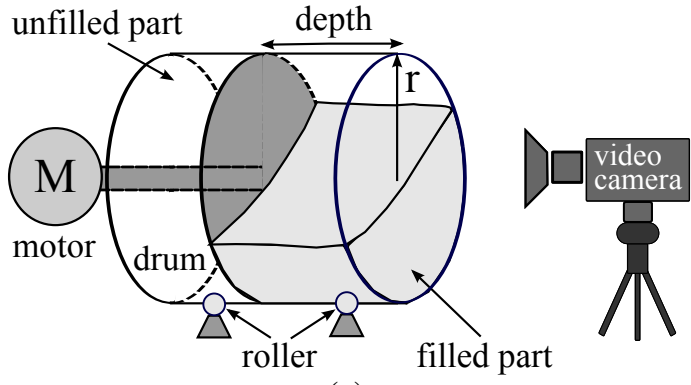

(a)

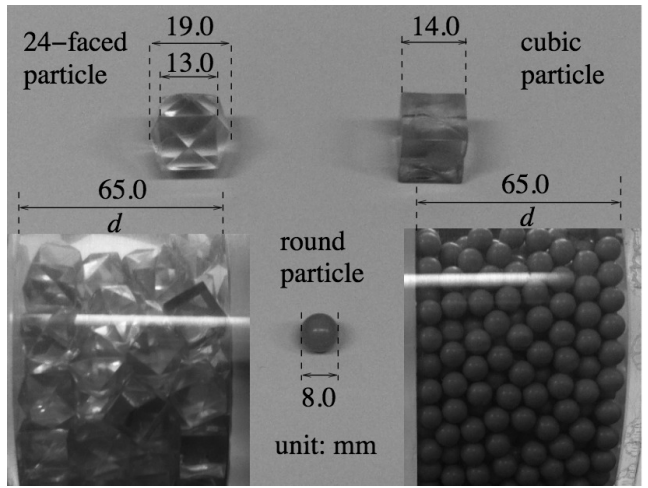

(b)

Figure 1. (a) Sketch of the experimental setup; (b) Particles used and snapshots along the depth direction.

mixture of 72 cubes and 135 particles with 24 faces were used for the large drum. A mixture of 38 cubes and 76 particles of 24 faces were used for the small drum.

Depends on the drum dimensions and rotation speed, the granular avalanches can be discrete or continuous $[6,7]$. To admit discrete avalanches of polyhedral particles, we kept the rotation speed $\Omega$ relatively slow, as $\pi / 6 \pm 0.01, \pi / 4 \pm 0.01$ and $\pi / 3 \pm 0.01[\mathrm{rad} / \mathrm{s}]$ (or $5 \pm 0.1$, $7.5 \pm 0.1,10 \pm 0.1[\mathrm{rpm}]$ respectively). Note that those speeds are a few orders higher than those admitting a discrete avalanche of spherical particles as reported in $[6,7]$. For sufficiently large rotation speeds, the dynamics is dominated by the rotation of the drum and the avalanches become continuous even for polyhedral particles. We took videos about 90 seconds long with a standard video format with a frame rate of 29.97 frames per second. For each case (combination of particle shape, drum size and rotation speed), four experiments have been conducted.

\subsection{Data processing}

The videos (mov-file) are taken in the axial direction so that the whole system is projected on the circular black backplate of the drum. The frames are then converted to successive images, e.g., as in Fig. 2 (a). Since the variation of the RGB values for the colored particles is much larger than the variation of the nearly pure color (black and white) of the background, the standard deviation of the RGB values of each pixel is used to distinguish the particles and the background. The background is then removed, see e.g., Fig. 2 (b). The center of gravity of the particles projected on the plane of view is then approximated as the averages of the coordinates in pixels. From

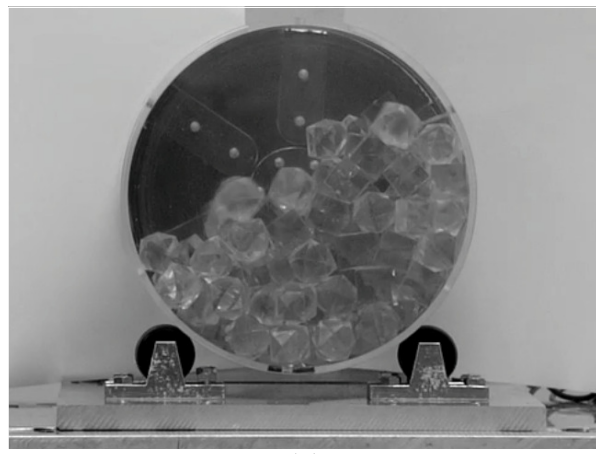

(a)

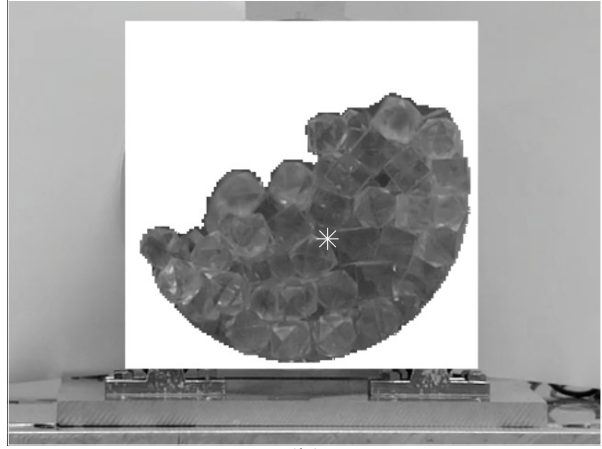

(b)

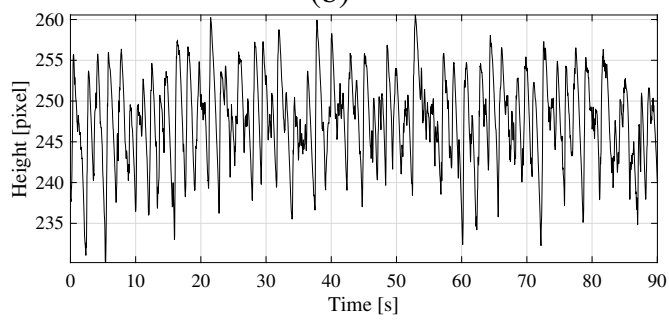

(c)

Figure 2. Image analysis: (a) One frame $(640 \times 480$ pixels $)$ from a video; (b) Image analysis to obtain the projected center of gravity of the particles, as the star mark; (c) A time series of the center of gravity in the height direction, with $\Omega=\pi / 6[\mathrm{rad} / \mathrm{s}]$.

the projected center of gravity from each frame, we obtain a time series of the vertical height, as shown in Fig. 2 (c).

Based on the time series, we compute the corresponding single-sided amplitude (SSA) spectrum using fast Fourier transform (FFT). Though ordinary power spectra from FFT would yield similar results, we present SSA spectra hereafter since they retain the same unit for the yaxis in a spectrum as the input signal being transformed. For a convenient comparison with the rotation speed, we scaled the horizontal axis of a spectrum from frequency $[\mathrm{Hz}]$ to angular frequency by multiplying $2 \pi$ [rad]. To show clearly the spectral characteristics, we present the SSA spectra plots both in linear and in log-log coordinates.

\section{Experimental results}

We focus on a novel indicator, the time series of the height of the projected center of gravity, instead of conventional slope angles. As will be shown, this indicator is also representative for granular avalanches. The underlying physics is that this indicator encloses directly the variation of the potential energy induced by drum motion and avalanches. 


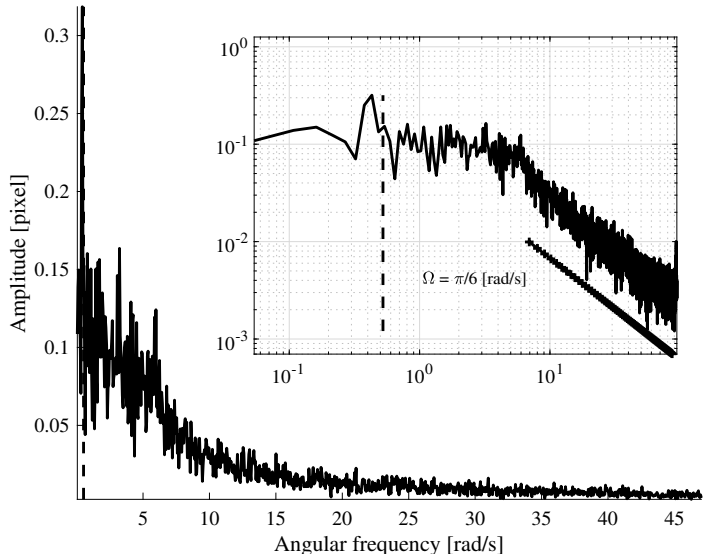

(a)

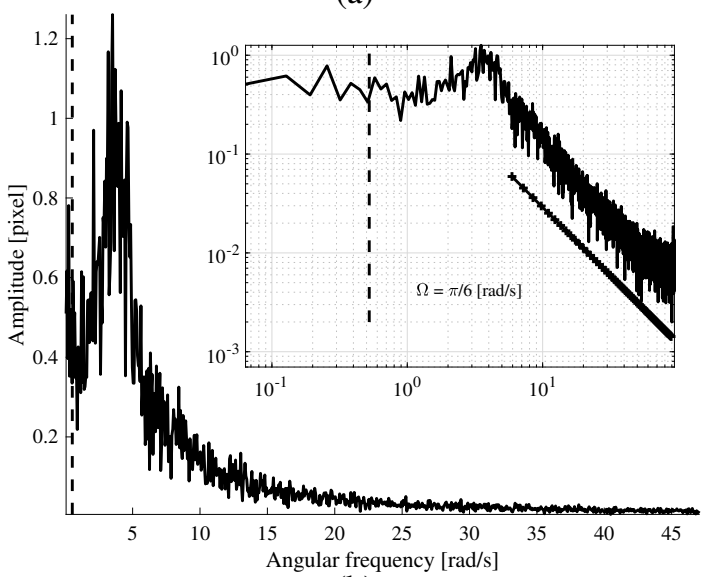

(b)

Figure 3. Influence of particle shape: (a) The SSA spectrum for spherical particles in the large drum with $\Omega=\pi / 6[\mathrm{rad} / \mathrm{s}]$; The shifted fitting line ('+' marker) for the $1 / f^{a}$-decay has a slope about $a=1.0$. (b) The SSA spectrum for polyhedral particles in the large drum with $\Omega=\pi / 6[\mathrm{rad} / \mathrm{s}]$; The shifted fitting line ('+' marker) for the $1 / f^{a}$-decay has a slope about $a=1.4$. The vertical dashed line indicates the rotation speed of the drum.

\subsection{Influence of particle shape}

Typical SSA spectra for spherical particles and polyhedral particles are shown in Fig. 3 (a) and (b) respectively. The differences between the two typical SSA spectra are threefold: First, a transition frequency range before the powerlaw decay for polyhedral particles is featured by a peak, which is is absent in the spectrum for spherical particles. Second, the spectrum for polyhedral particles decays faster than that for spherical particles, with a decay exponent $a=$ $1.37 \pm 0.07$ versus $a=1.04 \pm 0.02$. Third, there is a clear peak near the rotation frequency for the spherical particles which does not exist for the polyhedral particles.

The difference in the SSA spectra shows that the dynamics of the avalanches is influenced heavily by the particle shape. The existence of peak for polyhedral particles is a unique characteristic for the spectra of discrete avalanches. In contrast, the spectra for avalanches of spherical particles are featured by a plateau (or gradual decay) frequency region, indicating a continuous avalanche (flow) of surface layers. In the $1 / f^{a}$-power-law decay region, the avalanches of polyhedra have a faster decay rate than the $1 / f$ decay rate from the avalanches of spheres.

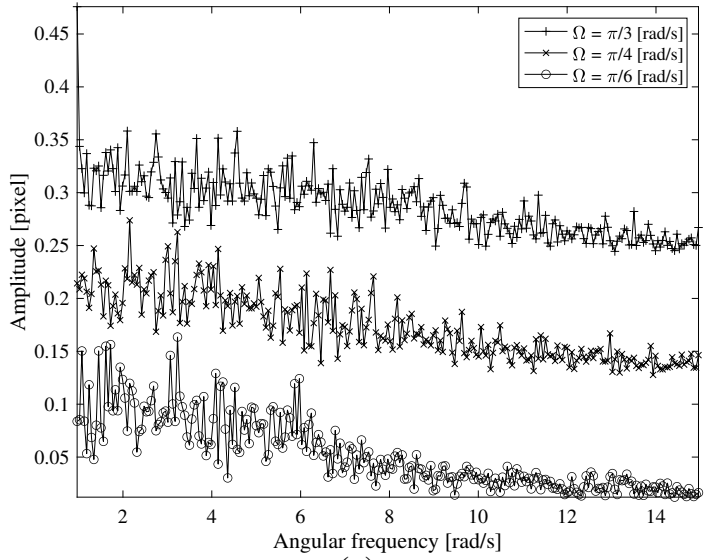

(a)

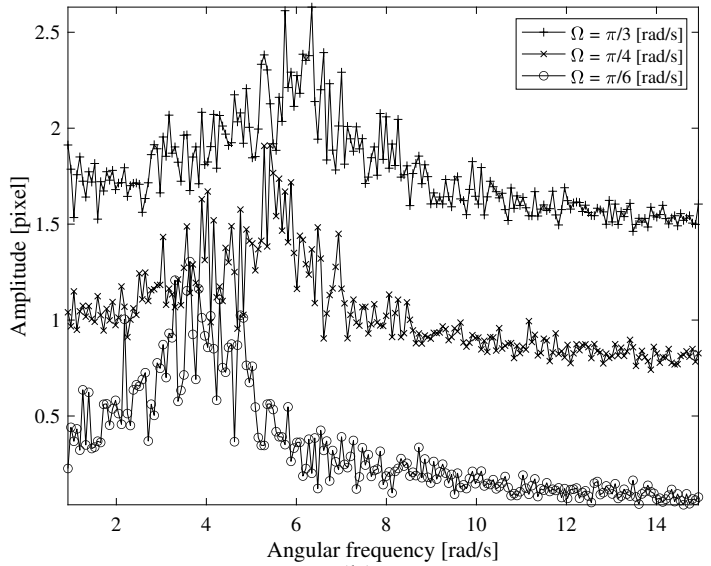

(b)

Figure 4. Influence of rotation speed on SSA spectra (the results for $\Omega=\{\pi / 4, \pi / 3\}[\mathrm{rad} / \mathrm{s}]$ were offset for legibility): (a) spherical particles and (b) polyhedral particles in the large drum.

\subsection{Influence of rotation speed}

Since the characteristic peaks for discrete avalanches of polyhedral particles are in a relatively low frequency range, we plot the SSA spectra of the three rotation speeds up to $15[\mathrm{rad} / \mathrm{s}]$ in the same figure, Fig. 4 (b). For better readability, the power spectra for the rotation speed of $\pi / 4$ and of $\pi / 3[\mathrm{rad} / \mathrm{s}]$ are shifted upwards. As can be seen, the frequency corresponding to a peak increases with the increase of rotation speed. The peaks are at about 6 to 7 times of the rotation frequency of the drum. In comparison, the results for the spherical particles show no peak in the same frequency range and the decay rate is not affected by the rotation speed, as shown in Fig. 4 (a). In terms of the angle of marginal stability, the peaks correspond to angles about $2 \pi / 7$ to $\pi / 3$. The rotation speed positively affects the location of the peaks in the SSA spectra for polyhedral avalanches while show no significant influence on the spectra for avalanches of spherical particles.

\subsection{Limitation and strength of system size}

We admit that the system sizes being studied are small. But we should also point out that the number of particles, though limited, are large enough to admit statistically meaningful results. As can be seen from Fig. 5 (a) and (b) for $\Omega=\pi / 3[\mathrm{rad} / \mathrm{s}]$, the SSA spectra for the two drums are 


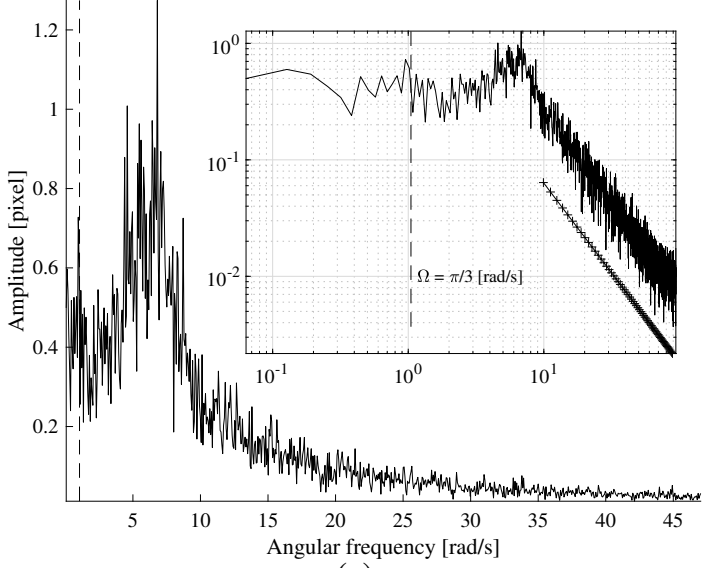

(a)

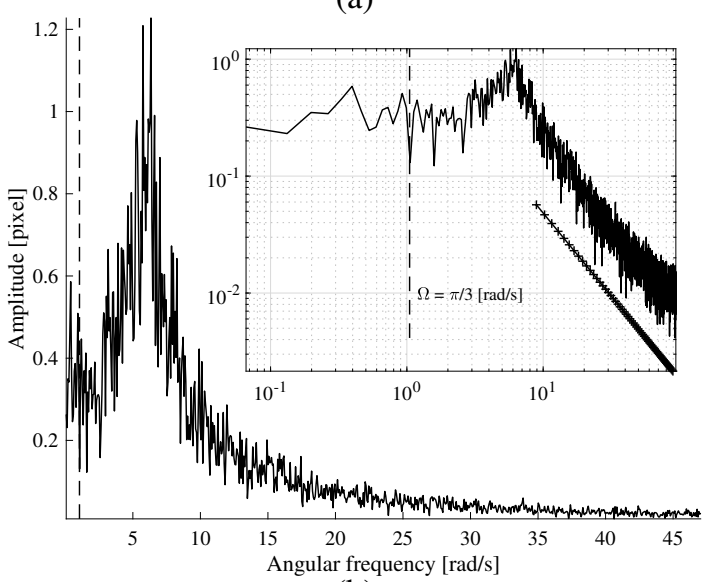

(b)

Figure 5. Influence of drum size: (a) The SSA spectrum for polyhedral particles in the small drum with $\Omega=\pi / 3[\mathrm{rad} / \mathrm{s}]$; The shifted fitting line ('+' marker) for the decay has a slope about $a=1.5$. (b) The SSA spectrum for polyhedral particles in the large drum with $\Omega=\pi / 3[\mathrm{rad} / \mathrm{s}]$; The shifted fitting line ('+' marker) for the decay has a slope about $a=1.4$. The vertical dashed line indicates the rotation speed of the drum.

very similar. Both spectra have distinct peaks corresponding to the dominant frequencies of discrete avalanches. The decay of the tail of the spectrum for the small drum is slightly faster than that of the large drum. For the other rotation speeds, the decay exponent for the small drum is $a=1.53 \pm 0.04$ and is $a=1.41 \pm 0.06$ for the large drum. These decay exponents for the polyhedral particles are far from $a=1.04 \pm 0.02$ for the $1 / f$-decay of the spherical particles. No qualitative difference is observed for the two drums. This proves that the system sizes studied are large enough to capture the avalanche dynamics inside drums.

The influence of the filling depth along the axial direction is another factor need to be considered. In the current settings, a large influence from the two parallel walls would increase the angle of marginal stability and consequently reduce the frequency of avalanches. In the future, larger drums with smaller particles and varying filling depth, need to be investigated to quantify the system-size dependent peak frequency and decay rate.

A strength of the small system sizes should be noted: the experimental results could serve as reference data to validate computer simulations of granular materials, without a limitation by the computational resources.

\section{Conclusions}

In this study, we investigated the avalanches of spherical and non-spherical granular particles inside half-filled rotating drums, by the SSA spectrum of the time variation of the center of gravity. We show that the spectra are characteristic with respect to the shapes of the particles. In the low frequency range of the spectrum, it has a peak for the discrete avalanches of polyhedral particles. There is no peak for the continuous avalanches of spherical particles. The high frequency tails are characterized by a power law decay $1 / f^{a}(a>0)$. A $1 / f$-decay is found only for spherical particles. For non-spherical particles the exponent significantly deviates from $a=1.0$. We conclude that there is no scale-invariant structure in general for granular avalanches, since the $1 / f$ spectrum is only observed for spherical particles. The spectral features presented in this paper could serve as reference data to validate computer simulations, considering the small number of particles and the regularity of the particle shapes being used.

\section{Acknowledgement}

The authors thank former students Tomohiro Matsuyama and Eiji Seo for their contribution to the experiments.

\section{References}

[1] P. Bak, C. Tang, K. Wiesenfeld, Phys. Rev. Lett. 59, 381 (1987)

[2] W.H. Press, Comments astrophys. 7, 103 (1978)

[3] E. Milotti, ArXiv Physics e-prints: physics/0204033 (2002)

[4] H.M. Jaeger, C.h. Liu, S.R. Nagel, Phys. Rev. Lett. 62, 40 (1989)

[5] V. Frette, K. Christensen, A. Malthe-Sorenssen, J. Feder, T. Jossang, P. Meakin, Nature 379, 49 (1996)

[6] J. Rajchenbach, Phys. Rev. Lett. 65, 2221 (1990)

[7] N.J. Balmforth, J.N. McElwaine, Granul. Matter 20, 1 (2018)

[8] O. Pouliquen, J. Delour, S.B. Savage, Nature 386, 816 (1997)

[9] J. Rajchenbach, Phys. Rev. Lett. 88, 014301 (2001)

[10] R. Fischer, P. Gondret, B. Perrin, M. Rabaud, Phys. Rev. E 78, 021302 (2008)

[11] M. Tischer, M.I. Bursik, E.B. Pitman, J. Sediment. Res. 71, 355 (2001)

[12] H. Yang, R. Li, P. Kong, Q.C. Sun, M.J. Biggs, V. Zivkovic, Phys. Rev. E 91, 042206 (2015)

[13] H. Yang, B. Zhang, R. Li, G. Zheng, V. Zivkovic, Powder Technol. 311, 439 (2017)

[14] Q. Chen, H. Yang, R. Li, W. Xiu, R. Han, Q. Sun, V. Zivkovic, Powder Technol. 364, 1039 (2020)

[15] N. Gaudel, S. Kiesgen de Richter, N. Louvet, M. Jenny, S. Skali-Lami, Phys. Rev. E 94, 032904 (2016) 